\title{
Hashimoto encephalopathy with high plasma monoamine metabolite levels: a case report
}

This article was published in the following Dove Press journal:

Neuropsychiatric Disease and Treatment

7 April 2017

Number of times this article has been viewed

\author{
Sho Horikoshi ${ }^{1,2}$ \\ Itaru Miura ${ }^{2}$ \\ Yasuto Kunii ${ }^{3}$ \\ Satoko Asano ${ }^{4}$ \\ Keiko Kanno-Nozaki² \\ Hirobumi Mashiko ${ }^{5}$ \\ Hirooki Yabe² \\ 'Department of Neuropsychiatry, \\ Hoshi General Hospital, \\ ${ }^{2}$ Department of Neuropsychiatry, \\ Fukushima Medical University \\ School of Medicine, ${ }^{3}$ Department \\ of Neuropsychiatry, Fukushima \\ Medical University School of Aizu \\ Medical Center, ${ }^{4}$ Department of \\ Neuropsychiatry, Japan Red Cross \\ Fukushima Hospital, ${ }^{5}$ Department \\ of Neuropsychiatry, Fukushima \\ Prefectural General Rehabilitation \\ Center, Fukushima, Japan
}

\begin{abstract}
Hashimoto encephalopathy (HE) is believed to be an immune-mediated disorder associated with Hashimoto's thyroiditis. It was suggested that neuropsychiatric symptoms, the presence of antithyroid antibody, and good response to steroids were important for the diagnosis of HE. It has been reported that homovanillic acid (HVA) and 3-methoxy-4-hydroxyphenylglycol (MHPG), which are monoamine metabolites of dopamine and noradrenaline, respectively, are the possible biomarkers of neuropsychiatric diseases. We report a case of Hashimoto encephalopathy, in which we longitudinally measured the plasma levels of monoamine metabolites. A 52-year-old woman developed acute psychosis, and was admitted to the psychiatric ward of our hospital due to psychotic state, 6 days after a traffic accident. An extensive evaluation showed no remarkable findings, except an increase in antithyroglobulin antibodies. Plasma levels of HVA and MHPG were extremely high at 66.5 and $41.8 \mathrm{ng} / \mathrm{mL}$, respectively. On day $16,50 \mathrm{mg}$ /day oral prednisolone was administered, which improved her psychotic symptoms. Plasma levels of HVA and MHPG decreased to 7.2 and $9.9 \mathrm{ng} / \mathrm{mL}$, respectively, on day 19. After the temporary worsening of psychosis and increase in plasma levels of HVA and MHPG, the dosage of prednisolone was tapered and low-dose risperidone was started. Her psychiatric symptoms gradually improved and plasma monoamine metabolite levels decreased again (HVA: $17.9 \mathrm{ng} / \mathrm{mL}$; MHPG: $7.7 \mathrm{ng} / \mathrm{mL}$ ). Although autoimmune mechanism has been suggested to be involved in HE, neural mechanism and pathogenesis of HE remain unknown. Our findings suggest that monoaminergic neural activity might be associated with psychotic symptoms in patients with $\mathrm{HE}$ and plasma levels of monoamine metabolites might be useful as state markers.
\end{abstract}

Keywords: Hashimoto encephalopathy, monoamine metabolite, homovanillic acid, 3-methoxy4-hydroxyphenylglycol, symptomatic psychosis

\section{Introduction}

Hashimoto encephalopathy (HE) is believed to be an immune-mediated disorder associated with Hashimoto's thyroiditis. In 1966, Brain et al described a 49-year-old man with Hashimoto's disease, who had various neuropsychiatric symptoms. ${ }^{1}$ In 1991 , Shaw et al described five patients with encephalopathy who responded to steroid, and reported that the activity of encephalopathy was associated with high antithyroid antibody titers. ${ }^{2}$ They called the encephalopathy "Hashimoto encephalopathy", and suggested that neuropsychiatric symptoms, presence of antithyroid antibody, and good response to steroids were important for the diagnosis of HE. Clinical diagnosis of $\mathrm{HE}$ is important, but difficult because HE includes various neuropsychiatric symptoms such as disturbance of consciousness, hallucinations, delusions, cognitive dysfunction, and personality changes. Moreover, there are few biomarkers for diagnosis of HE except for antithyroid antibody, and its underlying biologic basis remains unclear.
Correspondence: Itaru Miura Department of Neuropsychiatry, Fukushima Medical University, School of Medicine, Hikarigaoka I, Fukushima 960-1295, Japan

$\mathrm{Tel}+8 \mid 24547$ | 33 |

Fax +8I 245486735

Email itaru@fmu.ac.jp 
Homovanillic acid (HVA) and 3-methoxy-4-hydroxyphenylglycol (MHPG) are major metabolites of dopamine and noradrenaline, respectively. Plasma levels of HVA and MHPG were the possible biomarkers for psychiatric disorders including schizophrenia and delirium, ${ }^{3}$ and the changes in plasma monoamine metabolite levels were associated with the response to antipsychotics. ${ }^{3,4}$ Here, we report a case of $\mathrm{HE}$, in which we longitudinally measured the plasma levels of monoamine metabolites. In our department, we longitudinally measured plasma levels of monoamine metabolites in psychiatric inpatients using high-performance liquid chromatography, and this protocol was approved by the ethics committee of Fukushima Medical University. Written informed consent was obtained from the patient to publish this article.

\section{Case report}

A 52-year-old woman with faithful and mild personality who has never had medical or psychiatric history and drug/alcohol abuse and dependence was admitted to orthopedic hospital for neck pain after a traffic accident. She was diagnosed with contusion of neck and received painkillers. Three days after the traffic accident, she developed delusions, visual and auditory hallucinations, and her speech became incoherent. Although she was voluntarily discharged from the hospital after 5 days of admission, hallucinations and delusions lasted and she became agitated and violent. She was admitted to the psychiatric ward of our hospital due to psychotic state with disorientation and agitation, 6 days after the traffic accident. On admission (day 1), her physical and neurologic examinations (brain computed tomography, usual blood tests including levels of serum electrolytes, fasting blood glucose, and renal and liver function) showed no remarkable findings. But considerable changes were observed in the plasma levels of HVA and MHPG on admission; the level of HVA was $66.5 \mathrm{ng} / \mathrm{mL}$ (normal range for 50-60-year-old subjects, $4.0-15 \mathrm{ng} / \mathrm{mL}$ ) and the level of MHPG level was $41.8 \mathrm{ng} / \mathrm{mL}$ (normal range, $4.0-7.0 \mathrm{ng} / \mathrm{mL}$ ). Brain magnetic resonance imaging and whole-body computed tomography, electrocardiogram, and chest X-ray were all normal. Thyroid stimulating hormone and free $\mathrm{T} 3$ were normal, but free $\mathrm{T} 4$ was slightly elevated with $1.79 \mathrm{ng} / \mathrm{mL}$ (normal range, thyroid stimulating hormone: $0.50-5.00 \mu \mathrm{IU} / \mathrm{mL}$; free T3: $2.30-4.00 \mathrm{pg} / \mathrm{mL}$; free T4: $0.90-1.70 \mathrm{ng} / \mathrm{mL}$ ). Thyroid autoantibodies tests indicated normal titrates of antithyroid peroxidase antibodies $7.7 \mathrm{IU} / \mathrm{mL}$ (normal range, $0.0-15.9 \mathrm{IU} / \mathrm{mL}$ ), but elevation in titrates of antithyroglobulin antibodies (anti-TG) $92.3 \mathrm{IU} / \mathrm{mL}$ (normal range, $0.0-27.9 \mathrm{IU} / \mathrm{mL}$ ).

The clinical course after admission is summarized in Figure 1. A diagnosis of HE was suspected because of the

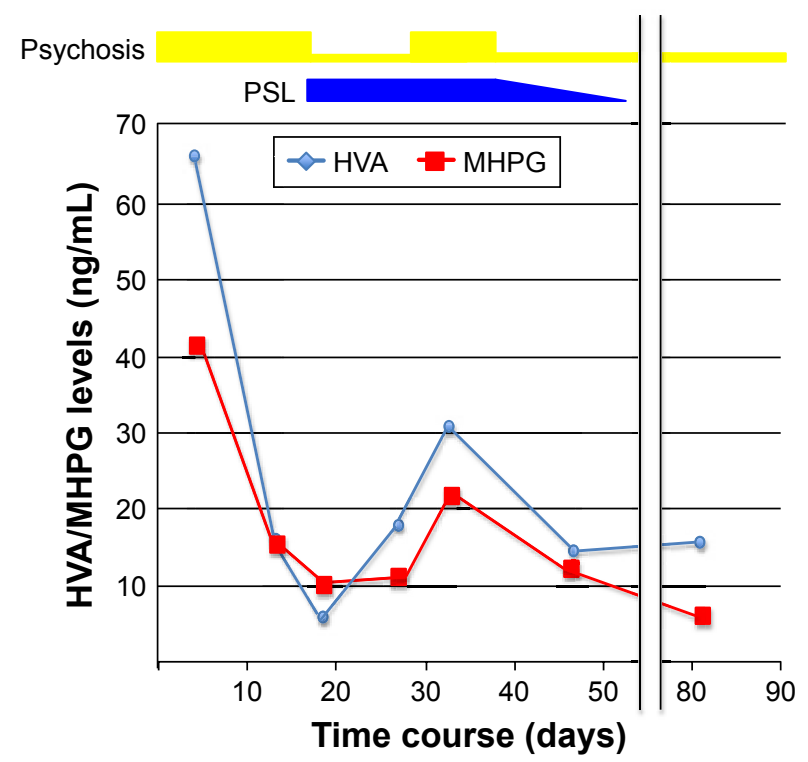

Figure I Clinical course and plasma levels of monoamine metabolite after administration.

Abbreviations: HVA, homovanillic acid; MHPG, 3-methoxy-4-hydroxyphenylglycol; PSL, prednisolone.

extensive negative work-up, disturbance of consciousness and psychotic symptoms, and positive anti-TG. On day 16, oral prednisolone (PSL) $50 \mathrm{mg} /$ day was administered. Disorientation, hallucinations, and delusions dramatically improved after the administration of PSL, and plasma levels of HVA and MHPG decreased to 7.2 and $9.9 \mathrm{ng} / \mathrm{mL}$, respectively, on day 19 . On day 27 , hallucinations and delusions appeared again, and plasma levels of HVA and MHPG increased to 33.1 and $22.4 \mathrm{ng} / \mathrm{mL}$, respectively, on day 33. After that, since a diagnosis of steroid psychosis was suspected, the dosage of PSL was decreased to $45 \mathrm{mg}$ /day and risperidone was started at $2 \mathrm{mg} /$ day. Subsequently, her hallucinations and delusions were alleviated. PSL was tapered, and her psychiatric symptoms gradually improved. On day 82 , plasma levels of HVA and MHPG were 17.9 and $7.7 \mathrm{ng} / \mathrm{mL}$, respectively.

\section{Discussion}

To our knowledge, this is the first case report where plasma levels of monoamine metabolites were measured in a patient with HE. In this patient, high plasma monoamine metabolite levels were observed with psychotic symptoms such as delusion and hallucination. The clinical features and the findings in titrates of anti-TG met both the criteria of Shaw et $\mathrm{al}^{2}$ and Peschen-Rosin et $\mathrm{al}^{5}$ for HE, leading to the diagnosis of HE, although we could not rule out the possibility of concussion because she developed psychosis symptoms after the traffic accident. Additionally, it is difficult to differentiate this HE from encephalopathy associated with autoimmune thyroid 
disease which is also called Hashimoto encephalopathy, a rare condition that occurs in patients with clinical or subclinical autoimmune thyroid disease. ${ }^{6}$

Notably, extremely high plasma levels of HVA and MHPG were observed, and these levels changed in parallel with the clinical course of psychotic symptoms, indicating that hyperactivity of dopamine and noradrenaline was associated with the psychotic symptoms in this case. This is consistent with a previous study suggesting that plasma HVA and MHPG levels are associated with psychotic or excitement symptoms in patients with schizophrenia.? Moreover, a previous report ${ }^{8}$ has demonstrated that plasma levels of HVA and MHPG were increased in systemic lupus erythematosus patients with psychiatric symptoms, which is consistent with our findings. The findings in this case suggest that dopaminergic and noradrenergic functions might play a role in the development of psychotic symptoms in patients with HE, and plasma levels of HVA and MHPG might be useful as state markers.

It has been reported that the monoaminergic neural system was involved in other symptomatic psychosis, such as hepatic encephalopathy and delirium. ${ }^{9-11}$ Patients with symptomatic psychosis have a lot of psychological symptoms, such as hallucination, delusion, mania, depression, dementia, and disturbance of consciousness. However, much of the mechanism and pathogenesis of symptomatic psychosis remains unknown. The possible causes of symptomatic psychosis including HE may be associated with abnormalities of the monoaminergic neural transmission, particularly in patients with psychotic symptoms like this case.

In summary, extremely high plasma levels of HVA and MHPG were observed in a case with HE. Dopaminergic and noradrenalinergic neural activity might be associated with psychotic symptoms in patients with HE or other symptomatic psychosis. Plasma levels of HVA and MHPG are possible biomarkers of psychosis and may also be useful as indicators of treatment response to antipsychotics.
Further studies with a large sample size are needed to confirm and extend these findings.

\section{Disclosure}

The authors report no conflicts of interest in this work.

\section{References}

1. Brain L, Jellinek EH, Ball K. Hashimoto's disease and encephalopathy. Lancet. 1966;2(7462):512-514.

2. Shaw PJ, Walls TJ, Newman PK, Cleland PG, Cartlidge NE. Hashimoto's encephalopathy: a steroid-responsive disorder associated with high antithyroid antibody titers-report of 5 cases. Neurology. 1991;41(2 (Pt 1)): 228-233.

3. Miura I, Takeuchi S, Katsumi A, et al. Effects of aripiprazole and the Taq1A polymorphism in the dopamine $\mathrm{D} 2$ receptor gene on the clinical response and plasma monoamine metabolites level during the acute phase of schizophrenia. J Clin Psychopharmacol. 2012; 32(1):106-109.

4. Yoshimura R, Ueda N, Shinkai K, Nakamura J. Plasma levels of homovanillic acid and the response to risperidone in first episode untreated acute schizophrenia. Int Clin Psychopharmacol. 2003;18(2):107-111.

5. Peschen-Rosin R, Schabet M, Dichgans J. Manifestation of Hashimoto's encephalopathy years before onset of thyroid disease. Eur Neurol. 1999;41(2):79-84.

6. Bharadwaj B, Sugaparaneetharan A, Rajkumar RP. Graves' disease presenting with catatonia: a probable case of encephalopathy associated with autoimmune thyroid disease. Acta Neuropsychiatr. 2012; 24(6):374-379.

7. Watanabe K, Miura I, Kanno-Nozaki K, et al. Associations between five-factor model of the Positive and Negative Syndrome Scale and plasma levels of monoamine metabolite in patients with schizophrenia. Psychiatry Res. 2015;230(2):419-423.

8. Ikenouchi A, Yoshimura R, Ikemura N, Utsunomiya K, Mitoma M, Nakamura J. Plasma levels of brain derived-neurotrophic factor and catecholamine metabolites are increased during active phase of psychotic symptoms in CNS lupus: a case report. Prog Neuropsychopharmacol Biol Psychiatry. 2006;30(7):1359-1363.

9. Bergeron M, Reader TA, Layrargues GP, Butterworth RF. Monoamines and metabolites in autopsied brain tissue from cirrhotic patients with hepatic encephalopathy. Neuroche Res. 1989;14(9):853-859.

10. Echizen H, Minegishi A, Hayashi S, Umeda N, Oda T, Ishizaki T. Raised plasma concentrations of 3-methoxy-4-hydroxyphenylethyleneglycol in cirrhotic patients with or without hepatic encephalopathy. Gut. 1989;30(5):656-664.

11. Nakamura J, Uchimura N, Yamada S, Nakazawa Y. Does plasma free3-methoxy-4-hydroxyphenyl(ethylene)glycol increase in the delirious state? A comparison of the effects of mianserin and haloperidol on delirium. Int Clin Psychopharmacol. 1997;12(3):147-152.
Neuropsychiatric Disease and Treatment

\section{Publish your work in this journal}

Neuropsychiatric Disease and Treatment is an international, peerreviewed journal of clinical therapeutics and pharmacology focusing on concise rapid reporting of clinical or pre-clinical studies on a range of neuropsychiatric and neurological disorders. This journal is indexed on PubMed Central, the 'PsycINFO' database and CAS,

\section{Dovepress}

and is the official journal of The International Neuropsychiatric Association (INA). The manuscript management system is completely online and includes a very quick and fair peer-review system, which is all easy to use. Visit http://www.dovepress.com/testimonials.php to read real quotes from published authors. 